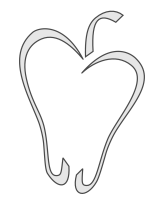

ISSN 1852-4249

\title{
Commentary on "Jamming transition in a two-dimensional open granular pile with rolling resistance"
}

\author{
Roberto Arévalo ${ }^{1 *}$
}

\section{Introduction}

The paper "Jamming transition in a twodimensional open granular pile with rolling resistance" studies the jamming transition using the flow of granular particles from piles. In this commentary I would like to give a perspective on the results by Magalhães et al. [1] and previous related studies obtained in silos.

We speak about jamming transition in a silo when an arch forms at the outlet effectively arresting the flow. This is likely to happen when the size of the outlet is a few times bigger than that of the flowing particles. What factors control the probability of arch formation? If we fix the nature of the particles, it is found that the pressure close to the outlet has a major influence. In Ref. [2], it was shown that altering this pressure in a two-dimensional silo can change the probability of arch formation by two orders of magnitude. At the same time, the flow rate or velocity field are modified only slightly.

In a flat-bottomed silo, pressure increases linearly from the free surface toward the bottom. At a certain depth, however, pressure saturates and remains constant until the base. This is the well known Janssen effect. It is usually attributed to

*E-mail: aroberto@ntu.edu.sg

1 Nanyang Technological University, School of Physical and Mathematical Sciences, Physics and Applied Physics, 21 Nanyang Link, 637371, Singapore. the friction of the walls that sustains part of the weight of the column of particles. The depth at which pressure saturates depends on properties of the grains (like the Poisson ratio) and the geometry of the silo.

This scenario for the silo contrasts greatly with that of a pile, as the ones studied in Ref. [1]. Piles have no lateral walls so no Janssen effect is present. Further, the geometry of the pile itself assures that pressure is not homogeneous. As it could be expected, pressure increases linearly from the exterior as we move toward the center. Surprisingly, the pressure is not maximum at the center of the pile. Instead, it shows a drop that has been termed "pressure dip" [3].

When one opens an orifice at the base of a silo, the material inside outpours driven by gravity. As stated, if the size of the outlet is a few times that of the flowing particles, an arch may form that jams the flow. As the size of the orifice grows, the probability of arch formation decreases. Eventually, for relatively large orifices, no arches are observed during the accessible time-scale and the flow seems continuous.

Actually, the last statement is currently under debate. Some authors have conjectured that there exist two regimes in the silo discharge. One for orifices with radius $R<R_{c}$, where $R_{c}$ is some critical value (in units of the particles' radii. Since most of the literature concerns disks in $2 D$ or spheres in $3 D$, I will refer here to the radii of the particles and that of the orifice). In this regime arching 
Papers in Physics, vol. 6, ART. 060008 (2014) / R. Arévalo

probability is always bigger than zero and arches are bound to appear. A second regime is claimed to exist for $R>R_{c}$, in which the probability to observe a stable arch is zero and the flow is actually continuous.

\section{Silos, piles and jamming}

Several works have been devoted in the last years to try to settle the accuracy of this picture, and to obtain the value of $R_{c}$ for different geometries. In Ref. [4], Zuriguel et al. experimentally study the case of a cylindrical silo filled with spherical particles as well as other shapes (small cylinders with low aspect ratio and rice). They measure the size $s$ of the avalanches, defined as the number of particles fallen between two clogs. It is found that their data are well described by a power law of the form

$$
<s>=A /\left(R_{c}-R\right)^{\gamma} .
$$

This leads them to propose that the avalanche size indeed diverges at $R_{c}$ (whose value depends slightly on the shape of the particles). For spheres, they find $R_{c}=4.94$ and $\gamma=6.9$.

A finite size analysis was carried out in Ref. [5]. To this end, the jamming probability function $J(N, R)$ was defined as the probability that the flow clogs before $N$ particles have fallen. In other words, the probability to observe an avalanche of size $N$ for a given aperture size $R$. Fixing the value of $N$, the authors compute the jamming probability $J_{N}(R)$. For low values of $N, J_{N}(R)$ presents a maximum value of 1 for low $R$ and a minimum value 0 for big aperture size. The transition between these values is fast but smooth, with a well defined slope. Upon increasing $N$, the transition becomes more abrupt and the slope steepens. A limiting step function as $N \rightarrow \infty$ would be the hallmark of a real abrupt transition separating the two regimes at $R_{c}$. The authors fit the data to $J_{N}(R)=\left\{1-\tanh \left[\alpha\left(R-R_{c}\right)\right]\right\} / 2$, which actually converges to the Heaviside function in the limit $\alpha \rightarrow \infty$. However, there is no theoretical justification for this expression, apart from the good fit.

An analogous research in $2 D$ (using spherical particles) was carried out in Ref. [6]. It was found that the data on avalanche size could be fit with the same expression than in the $3 D$ case, with values $R_{c}=8.5$ and $\gamma=12.7$. Nevertheless, a careful finite-size system analysis leads the authors to conclude that there are no two separated regimes in this case. As in the $3 D$ case, the jamming probability function $J(N, R)$ is used. Also in this case, the data from experiments show an apparent convergence toward a step function. However, by a subsequent theoretical analysis, the authors found a limiting function which is smooth and shows a doubly exponential dependence in $R$. The fact that the experimental data could be fit by Eq. (1) is explained taking into account that the maximum measured avalanche is for $R \simeq 5.5$ while the presumed critical value is much higher, $R_{c}=8.5$.

To et al. [7] have studied the jamming of disks in $2 D$ in a hopper geometry. Measuring the jamming probability defined above, they find that their data can be fitted with several different expressions. First, by an independent theoretical analysis of their experiment, an expression for $J_{N}(R)$ is found which is formally analogous to the one proposed by Janda et al., and does not contain any divergence. However, their data can be well fit by Eq. (1) and an exponential containing a divergent term. Being the curves indistinguishable in the range of data available, the conclusion is that the question of the existence of a critical outlet size cannot be settled.

Recently, Magalhães et al. have proposed a series of simulations $[1,8]$ addressing the existence of a critical outlet for the flow of grains in a different geometrical setting. They use a realistic soft particle molecular dynamics method with static friction, which is supplemented with rolling resistance in their contribution to this volume. Briefly, the protocol implemented is as follows: in $2 D$ a pile of grains is created by allowing particles to "rain" over a base of a certain width $L$, which is used as a measure of the size of the initial piles. Once the pile is stable, a hole of size $R$ is opened at the center of the base and particles flow through it. The pile ends up in one of two possible final states: i) a stable arch appears around the outlet blocking the flow; ii) no arch is formed and the pile collapses completely, only a few particles remain at both sides of the outlet. In both cases, the number of particles fallen is recorded and, in case i), also the height $h$ of the final pile.

The authors propose that this scenario can be 
Papers in Physics, vol. 6, ART. 060008 (2014) / R. Arévalo

likened to a phase transition between the two regimes. Taking $h$ as an order parameter, the plot $h(R)$ shows three regimes. For small apertures, $h$ is essentially a constant which depends on the size of the initial pile. For big $R$, the pile collapses and so $h=0$. The transition between the two values is gradual for small piles and becomes more abrupt for larger piles.

The fluctuations of $h$ as a function of the outlet size present a well defined peak at some value $R^{*}$ which depends on the size $L$ of the initial pile. As $L$ grows, $R^{*}$ seems to converge to a limiting value, and when extrapolating $L \rightarrow \infty$, the authors find a critical outlet size $R_{c}=5.0$.

In this case, there is no attempt at a theoretical analysis of the observations that could further support the extrapolation of $h(R)$ to a step function.

In their contribution to this volume, the authors revisit this problem with new simulations in which they implement rolling friction, making them more realistic. The main conclusion is that the picture drawn in their first work holds, although a correction in the value of $R_{c}$ is introduced.

\section{A critical outlet?}

Let us, at this point, critically address the results summarized in the previous section. Regarding the $3 D$ silo, the conclusion that there exists a critical outlet size is based on the good fit of the data on avalanche size by Eq. (1). In principle, there is no reason precluding the idea that avalanches keep growing for large orifices, with the probability of arch formation decreasing without ever reaching zero. That this is not observed could be simply due to the limitations of the experimental setup. The observations were carried out keeping the height of material inside the silo constant. This is required to assure steady state conditions and, in particular, that there is always a Janssen effect present (if the height of the material decreases too much, pressure does not saturate). Under this conditions, it was simply not possible to refill the silo in order to measure avalanches bigger that a few million of grains.

From a more theoretical perspective, Eq. (1) might seem a bit unsatisfactory. In first place, coefficient $A$ lacks a fundamental interpretation, being just the size of the avalanche for an orifice of size
$R=R_{c}-1$. In second place, the value of the exponent $\gamma$ is rather high compared to what one usually finds in phase transitions; and this holds for both the $2 D$ and $3 D$ silos. Third, the avalanche size should approach zero as the outlet size approaches one, in units of the diameter of the particles.

As a matter of fact, other expressions can be found to fit the available data. For example $<$ $s>=A(R-1) e^{\left(B \cdot R^{C}\right)}$ gives a good fit with $A \simeq 3.4$, $B \simeq 0.04$ and $C \simeq 3.8$. This expression is not divergent and goes to zero as the outlet size approaches that of the particles. Although given without theoretical motivation, it shows that the present data are not enough to settle the question of the existence of a critical outlet size. A better understanding of the process of arch formation and how it is affected by the flow is required to gain insight into this problem.

In the case of $2 D$ silos we find, in addition to a wealth of experimental data and careful finite size study, a theoretical insight into the process of arching. In both the independent analysis by To and Janda et al. formally equivalent expressions for the jamming probability are found. This reinforces the conclusion that the avalanche size does not diverge and there is no critical outlet. However, one must consider i) that there are underlying asumptions in the theoretical considerations, and ii) that the available data can be well fit with diverging expressions. More (difficult to obtain) data for orifices similar to the putative critical size would be necessary to make a strong statement.

It is worth mentioning that the theoretical derivation of the jamming probability in Ref. [6] leads, when applied to a $3 D$ silo, to an expression that does not fit the corresponding experimental data. This could be due to some assumption being valid in $2 D$ but not in $3 D$. Or, one can also consider the possibility that there is no transition in two dimensions, and a critical outlet size appears in three dimensions. This is a perfectly possible scenario, as we know from other phase transitions whose existence depends on the dimensionality of the system.

Magalhães et al. propose an analogous study in a radically modified geometry. One can imagine that removes the lateral walls of a $2 D$ silo, then the column of particles collapses and ends up in a pile of certain width. Under these conditions, there is 
Papers in Physics, vol. 6, ART. 060008 (2014) / R. Arévalo

no Janssen effect, the pressure is not homogeneous and the dynamics could be very different from that of a silo. In any case, as mentioned in the introduction, the probability of arch formation could be drastically affected. Thus, it is not obvious that the conclusions reached for silos should hold in the case of piles.

Actually, the conclusion in the previous work, Ref. [8], is that there exists a critical outlet for the flow from piles. The obtained value $R_{c}=5$ is far below to $R_{c}=8.5$, reported by Janda $\mathrm{el}$ al. for the $2 D$ silo. Without taking into account the existence or not of a critical outlet, this could be an indication that the two setups are not comparable.

In their present contribution, Magalhães et al. report a correction to $R_{c}=5.3$ for a contact model that includes rolling resistance $(\mathrm{RR})$. This is reasonable, since particles interacting with RR should interlock more easily, leading to more stable arches. Nevertheless, this new $R_{c}$ value is still much smaller than the one found for the $2 D$ silo.

\section{Avalanche size and flow}

It may seem difficult to imagine the formation of an arch stable enough to arrest the flow in a silo with a large aperture. Effectively, it appears that the flow will sweep any incipient arch and will continue undisturbed. However, the flow is a tunable feature of the silo, known to vary with the square root of gravity, as manifested in experiments [9-11] and simulations [12].

Gravity can be modified in experiments up to a certain point, and it is always possible in simulations, so let us call $\Gamma$ to the imposed body force on the particles. One can imagine, then, that reduces $\Gamma$, thus making the flow slower, even for large apertures. Under these conditions, molecular dynamics simulations [12] lead to the conclusion that the size of the avalanches increases with the kinetic energy of the system. This is so because, when reducing the driving force, the particles in the outlet region have more time to dissipate their kinetic energy and form a stable structure that blocks the flow. It is then conceivable that one can have a $\Gamma$ small enough to allow the particles form a stable arch before being swept by the flow.

Should this picture be correct, there would not be a critical outlet size. Arches would simply be less likely to be stabilize as the flow was increased by increasing $R$. The observation of arches would be limited by the time window allowed by the experiments or simulations. In order to shed some light in the process of arching a low $\Gamma$, additional simulations with a variable outlet size are currently under way.

\section{Conclusions}

In this brief commentary, I have tried to give a perspective on recent results on the jamming of particle flows in silos and its relation with Magalhães et al. contribution to this volume. It is commonplace that silos get jammed. When the size of the aperture is not much larger than that of the particles, arches appear to block the flow. Upon increasing the size of the outlet, arches become scarcer. And for large orifices, they are not seen at all at accessible time scales. The observation of power-law relations between the size of the avalanches and the outlet size sparked the interest in considering a critical-like transition between two regimes: a jamming regime in which arches are bound to appear and block the flow, and a continuous regime in which there are no arches. Both regimes would be separated by a critical outlet size.

Magalhães et al. undertake an analogous research changing the conditions of the reservoir from which the particles flow. They use piles, which have open boundaries, and introduce new complications to be considered. If not transferable from one another, the results in piles and silos will widen our perspective on the clogging of granular particles.

Let me, very briefly, summarize my opinion on the existence of a critical outlet:

- Experimental data in $3 D$ silos are compatible with a transition from a jammed to a continuos flow regime at a certain value of the outlet. However, descriptions not involving a divergence are also possible. So far, we lack a theoretical frame that justifies one view over the other.

- Experimental data in $2 D$ silos are also compatible with both the existence and the absence of a critical outlet. Some theoretical insights may point toward a picture without a critical 
Papers in Physics, vol. 6, ART. 060008 (2014) / R. Arévalo

outlet. However, one should take into account that:

- These theoretical insights contain underlying assumptions.

- They are not always based on a complete understanding of the arching process.

- Simulations in Refs. $[1,8]$ on the flow from $2 D$ piles are compatible with the existence of a critical outlet. There is not a theoretical picture in which to understand the results yet.

- Due to the disparities in the conditions of the reservoir, it is not a priori clear that results in $2 D$ silos and $2 D$ piles should be comparable.

- Based on results of recent simulations in $2 D$, one can speculate that an arch can always block the outlet irrespective of the flow rate and, hence of $R$. For large $R$, these arches are just extremely unlikely.

Possible ideas to advance in the future could be:

- Obtain more experimental data for $R$ close to the transition in silos and piles. This could eliminate alternative expresions for the avalanche size.

- Carry out experiments in inclined silos to mimic reduced gravity.

- Investigate the dynamics of particles in the region close to the outlet. Here, simulations should be extremely useful, given that one has access to all the variables involved.

- Extend the study of piles to three dimensions and diverse conditions, as reduced gravity.

[1] C F M Magalhães, A P F Atman, G Combe, J G Moreira, Jamming transition in a twodimensional open granular pile with rolling resistance, Pap. Phys. 6, 060007 (2014).

[2] I Zuriguel, A Janda, A Garcimartín, C Lozano, $\mathrm{R}$ Arévalo and D Maza, Silo clogging reduction by the presence of an obstacle, Phys. Rev. Lett. 107278001 (2011).
[3] A P F Atman, P Brunet, J Geng, G Reydellet, P Claudin, R P Behringer, E Clément, From the stress response function (back) to the sand pile "dip", Eur. Phys. J. E 1793 (2005).

[4] I Zuriguel, A Garcimartín, D Maza, L A Pugnaloni and $\mathrm{J}$ M Pastor, Jamming during the discharge of granular matter from a silo, Phys. Rev. E 71051303 (2005).

[5] I Zuriguel, L A Pugnaloni, A Garcimartín, D Maza, Jamming during the discharge of grains from a silo described as a percolating transition, Phys. Rev. E 68030301 (2003).

[6] A Janda, I Zuriguel, A Garcimartín, L A Pugnaloni and D Maza, Jamming and critical outlet size in the discharge of a two-dimensional silo, Europhys. Lett. 8444002 (2008).

[7] K To, P Y Lai and H K Pak, Jamming of granular flow in a two-dimensional hopper, Phys. Rev. Lett. 8671 (2001); K To, Jamming transition in two-dimensional hoppers and silos, Phys. Rev. E 71060301 (2005).

[8] C F M Magalhães, J G Moreira and A P F Atman, Catastrophic regime in the discharge of a granular pile, Phys. Rev. E 82051303 (2010).

[9] W A Beverloo, H A Leniger, and J van de Velde, The flow of granular solids through orifices, Chem. Eng. Sci. 15260 (1961).

[10] C Mankoc, A Janda, R Arévalo, J M Pastor, I Zuriguel, A Garcimartín and D Maza, The flow rate of granular materials through an orifice, Gran. Matt. 9407 (2007).

[11] S Dorbolo et al., Influence of the gravity on the discharge of a silo, Gran. Matt. 15263 (2013).

[12] R Arévalo, I Zuriguel, D Maza, A Garcimartín, Role of driving force on the clogging of inert particles in a bottleneck, Phys. Rev. E 89 042205 (2014).

[13] A Janda, I Zuriguel and D Maza, Flow rate of particles through apertures obtained from Self-Similar density and velocity profiles, Phys. Rev. Lett. 108248001 (2012). 